\title{
Mechanical Stability of Polystyrene and Janus Particle Monolayers at the Air/Water Interface
}

\section{Supporting Information}

Jessica Lenis, ${ }^{1,2}$ Sepideh Razavi, ${ }^{1}$ Kathleen D. Cao, ${ }^{2}$ Binhua Lin, ${ }^{3}$ Ka Yee C. Le $e^{2}$, Raymond S. Tu, ${ }^{1}$ and Ilona Kretzschmar, ${ }^{1}$

${ }^{1}$ Department of Chemical Engineering, City College of City University of New York, New York, New York 10031, United States

${ }^{2}$ Department of Chemistry, Institute for Biophysical Dynamics, and James Franck Institute, The University of Chicago, Chicago, Illinois 60637, United States

${ }^{3}$ James Franck Institute and Center for Advanced Radiation Sources, The University of Chicago, Chicago, Illinois 60637, United States

Video S1 and Video S2 are taken with an Olympus bright field microscope with a 10x objective sitting atop a vibration isolation table and show microstructure during compression-expansion cycles for $2.4 \mu \mathrm{m}$ polystyrene and Janus polystyrene particle layers at the air/water interface. Videos are recorded with a charge-coupled-device (CCD) camera and shown at an 8-times faster speed. Video S3 is a section of a compression-expansion cycle recorded with a 50x objective illustrating subduction for a Janus particle monolayer during compression.

A summary of optical micrographs is provided in Figure S1 for both (A) plain and (B) goldcapped Janus particle $(2.4 \pm 0.1 \mu \mathrm{m})$ films during a compression-expansion cycle showing the structure of the particle films 
(a)

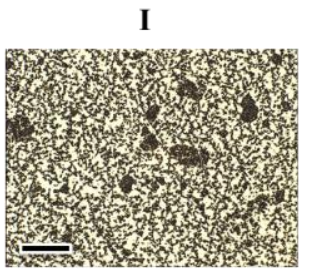

(b)

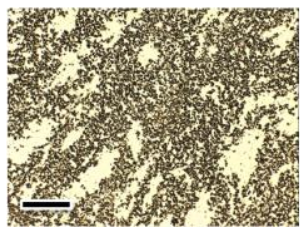

II
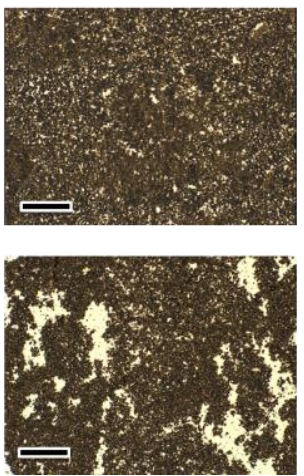

III
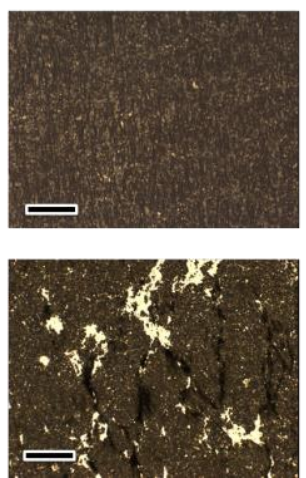

V
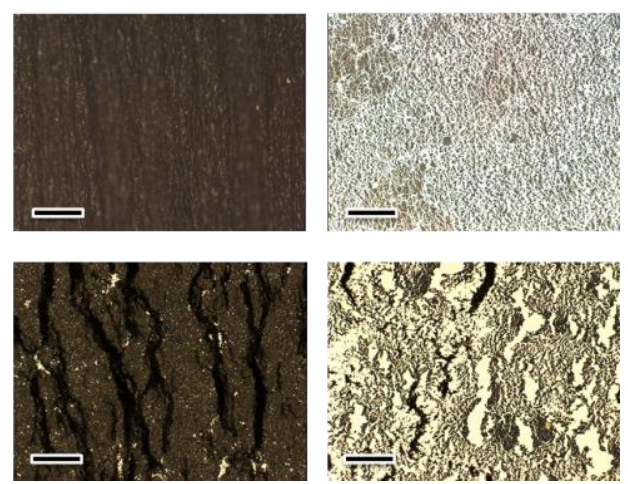

Figure S1. Optical micrographs of (a) plain and (b) gold-coated Janus polystyrene particles (2.4 $\pm 0.1 \mu \mathrm{m})$ at the air/water interface captured at different stages of compression and expansion: (I) after deposition, (II) close-packed network of particles, (III) onset of deformations out of the interfacial plane (striation and subduction), (IV) monolayer collapse (wrinkling and subduction), and (V) monolayer after expansion. Scale bar is $200 \mu \mathrm{m}$.

right after deposition (I), as a close-packed network (II), at the onset of out-of-plane deformation (III), during monolayer collapse (IV) and upon expansion (V).

Video S1 Description. Upon deposition, PS particles display some degree of clustering and aggregation, which is induced by capillary interactions. These clusters are uniformly distributed at the interface. Upon compression, a close-packed network of particles forms at the interface (see elapsed time 0:31) with an area packing fraction of $\phi=0.78 \pm 0.03$. Compression beyond this point gives rise to striation patterns that indicate the onset of out-of-plane deformations (see elapsed time 0:35). Further compression of the layer gives rise to the particle-layer collapse through wrinkling. Pronounced wrinkles can be readily observed at elapsed time 0:58. Wrinkling of the PS particle layer is a reversible process and the layer retains its 2D structure upon expansion (see elapsed time 1:34).

Video S2 Description. In contrast to the PS particle layer, where the particles form a network that uniformly spans the interface, Janus particles upon deposition form dendritic networks that 
are separated by particle-void areas. Compressing the layer gives rise to densification of the clusters, whereas the particle-void regions remain present at the interface (see elapsed time 0:26). Compressing the layers does not promote the merging of two clusters but the clusters slide by each other (see the lower right corner of the screen between elapsed times 0:30 and 0:34). When the compression leaves no more room for sliding, one cluster subducts under another cluster as they come into contact in a compressed state. The subduction occurs despite the particle-void areas that are present at the interface (see elapsed time 0:38). Further compression of the layer results in an increase of the area of the black patches. A scan of the trough area at maximum compression reveals that the subduction areas are spread uniformly throughout the interfacial film (1:30-1:53). Unlike the PS particle layer, the collapse of the Janus particle layer through subduction is an irreversible mechanism and upon full expansion the subducted areas remain unaltered (see elapsed time 2:30).

The pressure isotherms and microscopy images for $1 \mu \mathrm{m}$ plain polystyrene and gold-capped Janus polystyrene particle layers are shown in Figure S2. Particles are prepared using the same method described in the manuscript with the exception that sulfated polystyrene (PS) particles with a diameter of $0.960 \pm 0.016 \mu \mathrm{m}$ from Invitrogen are used. $300 \mu \mathrm{l}$ of a particle suspension with $10 \mathrm{mg} / \mathrm{mL}$ concentration in isopropanol/water is spread on an aqueous mixture of $1.985 \mathrm{M}$ $\mathrm{NaCl}$ and $0.4 \mathrm{M}$ glucose used as a density-matched subphase $\left(\rho_{\text {sub }}=1.088 \mathrm{~g} / \mathrm{cm}^{3}\right)$. A PTFE Langmuir trough (NIMA, UK) with an initial surface area of $78 \mathrm{~cm}^{2}$ is used to form the particle film at the air/water interface and to carry out isothermal compression and expansion measurements at $0.12 \mathrm{~mm} / \mathrm{s}$. The trough is housed on the stage of an Olympus bright field microscope with a 10x objective sitting atop a vibration isolation table, and images of the surface morphology of the particle film are recorded with a charge-coupled-device (CCD) camera. A 

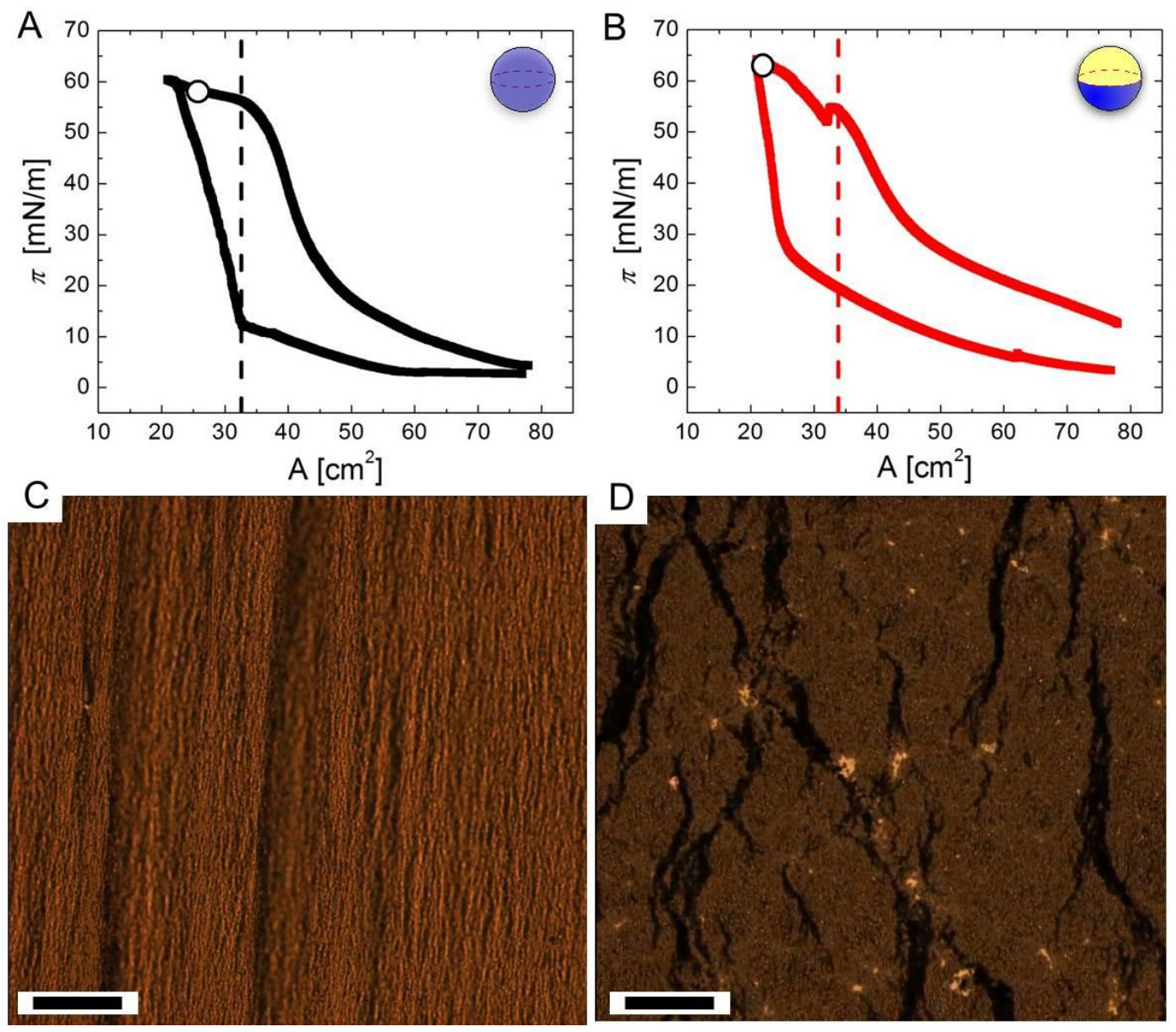

Figure S2. Compression-expansion isotherms of $0.960 \pm 0.016 \mu \mathrm{m}$ (A) plain and (B) goldcoated Janus polystyrene particle layer at an air/water interface. The two films show different collapse behaviors: optical microscopy image of (C) the plain polystyrene particle layer at a surface pressure of $58 \mathrm{mN} / \mathrm{m}$ shows buckling instability, whereas that of (D) the Janus particle layer at a surface pressure of $64 \mathrm{mN} / \mathrm{m}$ shows subduction instability. The dashed lines in the isotherms indicate the onset of layer instability in the two particle films; the circle in each isotherm identifies the pressure at which the corresponding image was taken. Scale bars are 100 $\mu \mathrm{m}$.

Wilhelmy plate oriented parallel to the barriers is used to measure the surface pressure. All experiments are performed at room temperature $\left(21-23{ }^{\circ} \mathrm{C}\right)$.

Inverse Langmuir-Schaefer Transfer Technique: ${ }^{1,2}$ Particle packing and orientation in the plain and gold-coated Janus polystyrene particle layers transferred from the air/water interface are investigated with scanning electron microscopy (SEM). After isothermal compression to the 
closed-barrier state, the respective particle layer is transferred from the Langmuir trough onto a silicon wafer substrate by an inverse Langmuir-Schaefer transfer technique similar to that reported by Lee et al., ${ }^{1}$ which is schematically shown in Figure S3. In brief, a cleaned silicon wafer substrate $(1 \times 1 \mathrm{~cm})$ is placed in a stainless steel apparatus with a surrounding $2 \mathrm{~mm}$ high machined knife edge. The entire setup is placed on the bottom of the trough prior to the experiment where it remains submerged in the subphase throughout the compression isotherm (Fig. S3A). At the closed-barrier state, the subphase is slowly aspirated from the trough (outside the barriers) to lower the subphase level without disturbing the particle layer, and the knife edge cuts the particle layer as the subphase level is lowered, preserving the particle layer morphology (Fig. S3B). Drilled holes in the bottom of the steel piece allow water to exit the chamber completely until the particle layer is deposited on the silicon wafer substrate (Fig. S3C). The substrate with the particle layers is then air dried and imaged with SEM.

A scanning electron micrograph of a $2.4 \mu \mathrm{m}$ polystyrene particle layer is shown in Figure S4. The film was deposited after full compression from the air-water interface onto a silicon wafer via the inverse Langmuir-Schaefer transfer method described above. 


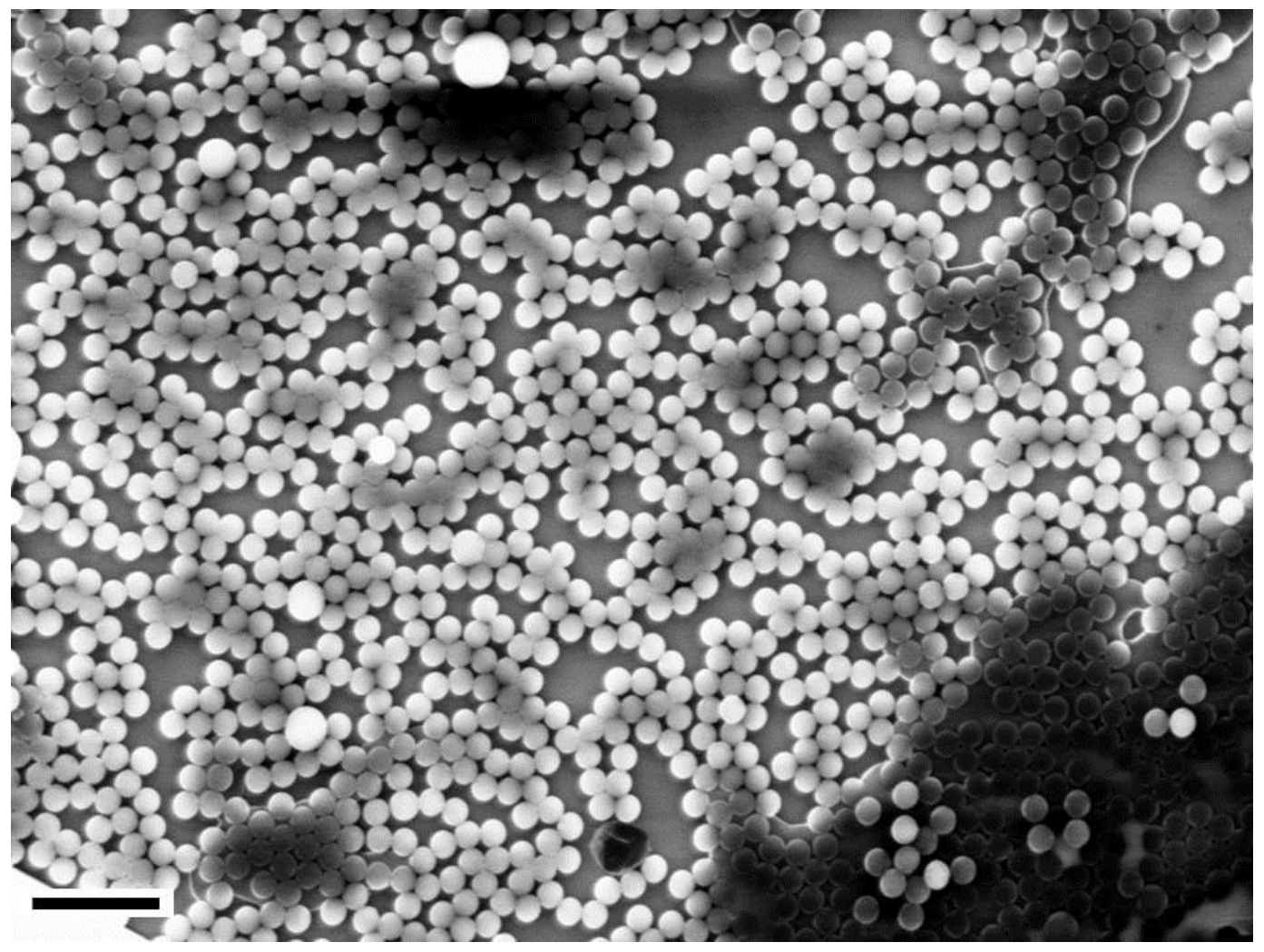

Figure S4. Scanning electron micrograph of a $2.4 \mu \mathrm{m}$ polystyrene particle layer deposited after full compression on a silicon wafer via the inverse Langmuir-Schaefer transfer method shown in Figure S3. The scale bar is $10 \mu \mathrm{m}$.

References:

(1) Lee, K. Y. C.; Lipp, M. M.; Takamoto, D. Y.; Ter-Ovanesyan, E.; Zasadzinski, J. A.; Waring, A. J. Apparatus for the Continuous Monitoring of Surface Morphology via Fluorescence Microscopy during Monolayer Transfer to Substrates. Langmuir 1998, 14, 2567-2572.

(2) Frey, S. L.; Chi, E. Y.; Arratia, C.; Majewski, J.; Kjaer, K.; Lee, K. Y. C. Condensing and Fluidizing Effects of Ganglioside GM1 on Phospholipid Films. Biophys. J., 94, 30473064. 